Les actes de colloques du musée du quai Branly Jacques Chirac

$7 \mid 2016$

Nathan Wachtel. Histoire et anthropologie

La conquête sans majuscule. L'affaire Diego de Avila (Manille-Mexico, 1577-1580)

\title{
Romain Bertrand
}

\section{(2) OpenEdition}

Édition électronique

URL : http://journals.openedition.org/actesbranly/692

ISSN : $2105-2735$

Éditeur

Musée du quai Branly Jacques Chirac

Référence électronique

Romain Bertrand, «La conquête sans majuscule. L'affaire Diego de Avila (Manille-Mexico,

1577-1580) ", Les actes de colloques du musée du quai Branly Jacques Chirac [En ligne], 7| 2016, mis en ligne le 15 novembre 2016, consulté le 08 septembre 2020. URL : http://journals.openedition.org/ actesbranly/692

Ce document a été généré automatiquement le 8 septembre 2020

(c) Tous droits réservés 


\title{
La conquête sans majuscule. L'affaire Diego de Avila (Manille- Mexico, 1577-1580)
}

\author{
Romain Bertrand
}

\section{Romain BERTRAND}

Merci à Serge et à tous ceux qui nous ont fait l'amitié d'assister à ces deux journées d'études. Tout d'abord, je dois dire que j'ai l'immense plaisir de participer ce jour à ce qui est non seulement un hommage rendu à Nathan WACHTEL, mais aussi une conversation nouée autour de son œuvre. Puisqu'il était question, dans le propos liminaire de Nathan WACHTEL, de fantôme, je me dois de dire à quel point j'ai été hanté par La Vision des vaincus, dont les thèses ont joué un rôle important dans mes propres réflexions tout au long de ma carrière.

2 En effet, $L a$ Vision des vaincus est, outre un titre d'une rare puissance d'évocation, un programme de recherche qui démontre aujourd'hui encore sa pleine actualité à travers un ensemble toujours plus riche de travaux consacrés à des situations de contact ou de conquête - des travaux qui ne sont plus limités, tant s'en faut, au domaine américaniste. Il s'agit par ailleurs d'un titre en forme de paradoxe, voire d'oxymore. Si les vaincus avaient eu "une vision", s'ils avaient préservé un horizon de pensée et d'espérance leur permettant de continuer à faire sens de leur quotidien par-delà l'effondrement démographique et la dévastation politique, alors ils n'étaient pas tout à fait des vaincus, ou du moins ils ne l'étaient pas tant que cela. Je crois que j'ai inscrit mes travaux dans le sillage de ce paradoxe.

Cette communication porte sur un monde qui sera probablement faussement familier aux américanistes, puisqu'il est l'un des points de projection les plus distants de la Monarchie hispanique, à savoir les Philippines du dernier quart du XVI siècle. Évidemment, il est beaucoup question du Mexique dans cette histoire, ou plutôt de cette Espagne largement diluée qui, depuis les côtes mexicaines, s'est lancée à l'assaut du Pacifique. Par bien d'autres aspects, toutefois, ce monde ressemble très peu au monde méso-américain, puisque nous sommes, au sens propre, au bord du monde 
malais-musulman, donc de l'Asie du Sud-Est insulaire. Je suis évidemment beaucoup plus familier de ce monde que celui de la Méso-Amérique.

Pour vous introduire à cette situation dite de " conquête espagnole des Philippines » à la fin $\mathrm{du} \mathrm{XVI} \mathrm{X}^{\mathrm{e}}$ siècle, je souhaiterais vous livrer une brève série de réflexions sur les dimensions de la conquête. Dans L'Amour et la justice comme compétences, le sociologue Luc BOLTANSKI commençait par une mise en garde en affirmant qu'il ne fallait pas énoncer au préalable les dimensions d'une "affaire ». Il fallait plutôt, chemin faisant, trouver dans le matériau lui-même les dimensions que les acteurs avaient imparties de façon contradictoire à l'objet. Avant de travailler sur une situation de conquête, nous devons donc tenter d'en prendre la mesure, d'en jauger la taille. Or il y a une tendance persistante, dans l'historiographie du "Pacifique des Ibériques", à unifier arbitrairement l'ensemble des opérations politiques, militaires et spirituelles qui y ont pris place sous l'égide des couronnes d'Espagne et du Portugal en dotant le terme de Conquête d'une majuscule assez pompeuse. D'une certaine façon, il s'agira de corroder cette majuscule au fil de l'exposé.

5 S'agissant de la conquête des Philippines, la période des commencements, celle qui court grosso modo de 1565 à 1590, m'intéresse tout particulièrement. En termes de taille, nous sommes confrontés à quelque chose de restreint. L'expédition de Miguel LOPEZ de LEGAZPI ouvre en 1565 la voie aux implantations militaires espagnoles dans l'archipel. Or, c'est une petite expédition, notamment si nous la comparons aux grandes expéditions de Floride ou de Californie des années 1550. Deux galions et deux pataches, cent cinquante marins et deux cents soldats: au regard des critères de l'époque, ce n'est pas une expédition de très grande envergure. S'agissant des effectifs militaires espagnols lors des campagnes dites de "pacification» de Cebu et de Panay, ces deux îles où s'implantent en premier lieu les Espagnols, il s'agit en tout et pour tout de trois cent soixante hommes. Ceux qui étaient à pied d'œuvre - et les pieds dans la boue dans les mangroves ne formaient rien de plus que quatre compagnies d'infanterie et une compagnie d'artillerie comptant chacune un maximum de quatre-vingts soldats. Parmi la soldatesque, les horizons d'interconnaissance sont donc particulièrement réduits : tous les vecinos de Manille ont bivouaqué ensemble au temps où ils étaient sous les ordres d'un sargento mayor. Le caractère modeste, sinon infinitésimal des effectifs perdure d'ailleurs longtemps. Projetons-nous en 1576, une dizaine d'années après le début de la conquête. Le nouveau gouverneur des lieux, Francisco de SANDE, qui vient tout juste d'arriver à Manille, estime, dans une lettre au Roi, qu'il n'y a pas plus de cinq cents Espagnols dans l'archipel. Il s'agit d'un archipel de dimensions conséquentes, qui court sur plus d'un millier de kilomètres, et nous pouvons en estimer la population à plusieurs centaines de milliers d'habitants, et même à deux ou trois millions selon certains démographes.

Soit un exemple un peu plus concret, celui de l'île de Cebu, où s'implantent en premier lieu les Espagnols. En 1572, Miguel Lopez de LEGAZPI y dénombre vingt-huit mille tributaires, ce qui est un chiffre probablement exagéré. Si nous le réduisons un peu et que nous appliquons le ratio multiplicateur ordinaire de 2.5 habitants pour 1 tributaire, nous arrivons à une population de quarante à cinquante mille habitants à une époque où le seul campement fortifié des Espagnols (la bourgade de Santísimo Nombre de Jesus) compte tout au plus quarante vecinos et une vingtaine de soldats d'active. Cinquante mille indigènes pour soixante Espagnols: on voit qu'il y a loin, ici, de la carte au territoire, c'est-à-dire de la pétition de souveraineté au contrôle effectif des sociétés 
et des ressources locales. Jusqu'à la fin du $16^{\text {ème }}$ siècle, la conquête se résume en réalité à une succession d'entradas aussi brèves que chaotiques, menées par des capitaines impétueux qui ne rendent pas toujours compte à la Couronne de leurs découvertes ni de leurs déconvenues. Tout cela aboutit à une domination de liseré, à un contrôle territorial limité à quelques segments côtiers d'un nombre modique d'îles des Philippines - essentiellement Cebu, Panay et la côte occidentale de Luçon.

Une fois établie la juste proportion des faits, une fois mieux cernée la manière dont les Espagnols ont pris les Philippines au moyen de leurs mousquets, il reste à s'interroger sur la façon dont les mondes locaux ont pris les Espagnols dans les rets de leurs modes de pensée. Rappelons en premier lieu qu'il y a au bas mot $30 \%$ de Mexicains parmi les effectifs des «flottes de secours » envoyées depuis la Nouvelle-Espagne aux Philippines entre 1566 et 1580. Un bon tiers des Espagnols vivant à Manille et à Cebu sont donc nés au Mexique, aussi bien dans la ville de Mexico que dans des régions plus excentrées et moins "pacifiées ", notamment au Jalisco et dans la région de Colima. A bord des nefs des armadas, il y a aussi des "nègres du Roi » c'est-à-dire des esclaves publics originaires de la Sénégambie et des pourtours du golfe de Guinée. Ainsi LEGAZPI débarque-t-il à Cebu, en avril 1565, en compagnie des "nègres " Juan de ALMONTE, Fernando BRAN, Pedro BIAFARA et Cristobal de BOREGADO, lesquels ont à charge de s'occuper de la maintenance des entrepôts royaux. Derrière le mot "Espagnols » se cache donc une réalité humaine on ne peut plus bigarrée.

J'aborde désormais l'essentiel de cette communication, dont les archives inquisitoriales forment l'armature documentaire. Les travaux de Nathan WACHTEL ont amplement démontré qu'il s'agissait d'une source d'informations tout à la fois dangereuse et précieuse. Je m'appuie ici sur une série de «causes » ouvertes par les Commissaires du Saint-Office aux Philippines dans la période 1577-1625, dont les actes sont conservés dans le ramo Inquisición de l'Archivo General de la Nación,au palais de Lecumberri, à Mexico. Au regard de ces documents, et donc du projet répressif qui les anime, nous voyons se dessiner trois situations de commerce coupable avec les savoirs rituels locaux.

9 Nous pourrions résumer un premier ensemble de dossiers sous la catégorie nécessairement vague de l'usage espagnol de magies du quotidien indigènes. Il s'agit d'affaires qui concernent en tout premier lieu des «soldats pauvres» qui n'avaient obtenu ni le droit de retourner en Nouvelle-Espagne une fois effectué leur service militaire, ni le bénéfice d'une encomienda. Certains, pour survivre, recevaient même un secours alimentaire de la Caisse royale, à savoir quelques fanègues de riz chaque mois, et beaucoup vivaient dans des cases de nipa, dans les quartiers les plus miséreux de Manille, loin de la plaza mayor que bordaient la cathédrale et le fort Saint-Jean. Nous trouvons ici un recours régulier aux onguents curatifs et aux potions de séduction préparés à base de plantes propres aux pharmacopées locales. Une plante en particulier, le lumay, sert à s'attirer des faveurs affectives et sexuelles. Par exemple, en 1625 , un élève du collège jésuite de Saint-Joseph à Manille, un garçon de bonne famille nommé Germano Roman Castañeda, avoue de son propre chef au commissaire du SaintOffice qu'il s'est procuré cette plante lors d'un séjour à Cebu, car il a entendu dire qu'elle est "efficace pour attirer à soi les femmes». Si le lumay permet d'éveiller ou d'attiser le désir d'un tiers, une autre plante, le talampunay, permet d'assouplir sa volonté, voire de le plonger dans un état d'hébétude. On peut ainsi en faire usage pour " apaiser » un mari un peu trop brutal. Cette plante, que les Espagnols et les Portugais 
des Indes appellent la "plante des cocus ", est en fait le datura, dont les effets euphorisants fascinent les Européens à compter de leur arrivée en Asie. Les missionnaires commettent ainsi des pages et des pages sur la façon dont les indigènes mettent en leur pouvoir les colons et les soldats au moyen de baumes ou de décoctions de talampunay. En 1617, une jeune métisse de vingt-quatre ans, Ybona de SUFINO, utilise le talampunay pour endormir son mari tandis que son "galant» (entendez son amant) lui rend visite à demeure.

10 Par ailleurs, les «soldats pauvres » recourent fréquemment à un ensemble de magies divinatoires qui sont d'autant plus efficaces qu'elles sont d'origine lointaine, c'est-àdire mises en œuvre par des praticiens d'origine africaine ou par des Chinois du quartier du Parian, situé dans les faubourgs extramuros de Manille. Dans cette Manille du tournant du $17^{\text {ème }}$ siècle, nous sommes bien dans le cadre d'une ville- mondes où se croisent, s'entrechoquent et s'entremêlent des bribes, des fragments de savoirs thérapeutiques venus de ce qu'on appelait à l'époque les "quatre parties du monde». Nous retrouvons en particulier deux techniques de divination : celle dite "du bilao", terme d'origine chinoise qui désigne une battée de bambou tressé de forme conique, et celle dite « des ciseaux », cette dernière pratiquée surtout par des " cafres», c'est-à-dire des Africains - lesquels sont soit des esclaves royaux venus de Nouvelle-Espagne avec les "flottes de secours", soit des marrons ayant fui les comptoirs portugais de l'Inde et des Moluques. Enfin, il existe tout un arsenal de sorts de bonne fortune et de protection. Il s'agit souvent de magies d'invulnérabilité, qui sont l'une des grandes constantes anthropologiques du monde malais-musulman à l'époque moderne. Les amulettes et les talismans d'invulnérabilité, appelés anting-anting ou agimat (un mot qui vient du malais jimat), sont ainsi très répandus dans les Visayas et à Luçon, et les soldats espagnols n'hésitent pas en acheter et à les porter de façon discrète sous leur chemise.

11 Mais à ces sorts défensifs s'ajoutent des sorts offensifs. Et, chose notable, nous n'avons plus seulement affaire, sous cette rubrique, à de simples soldats, mais aussi à des dames de la bonne société coloniale, des doñas mariées à des fonctionnaires royaux ou à de riches négociants qui ont fait fortune en chargeant à ras bord la nao de China (la nef Acapulco-Manille) de mantas de coton tissé ou de ballots de soie et de pièces de porcelaine chinoises. En mars 1627, une dame de Tondo, ville voisine de Manille, nommée doña Cathalina DE GUZMAN et qui est la maîtresse du capitaine Pedro ALVAREZ, loue ainsi pour deux cents tostones les services d'une Indienne appelée GALINGIN afin que celle-ci fasse périr son mari, le capitaine Juan de CAŇEDO, par voie de magies mauvaises. Nous basculons ici dans un second type de commerce coupable avec les savoirs indigènes que nous pourrions caractériser comme le recours à des spécialistes rituels locaux. En octobre 1611, Frère Antonio de PORRAS, le commissaire du Saint-Office à Manille, ouvre une instruction contre doña Maria de ZALDIVAR, qu'il accuse de s'être adjoints, pour la somme de cinquante pesos, les services de deux Indiennes, Catalina LIMABAN et Catalina TALOQUI, lesquelles témoignent longuement au fil du procès. Si doña Maria a requis l'assistance des deux Indiennes, c'est qu'elle souhaite faire périr une rivale en amour, doña Magdalena, l'épouse d'un personnage assez important dans la petite société maniléenne de l'époque puisqu'il s'agit du secretario de gobernacion de l'Audience de Manille, c'est-à-dire du premier greffier du tribunal royal de la ville, Pedro HURTADO de ESQUIVEL. A la demande de doña Maria, 
les deux Indiennes organisent une cérémonie d'invocation des esprits que les sources inquisitoriales désignent au moyen du terme de maganito.

La raison pour laquelle le Commissaire du Saint-Office prend un intérêt tout particulier à cette affaire est que la commanditaire, doña Maria, a pris part en personne à ce rituel. Elle a dansé frénétiquement au son des gongs de bronze et de laiton, munie d'un sabre japonais (katana), et ce afin de hâter l'ensorcellement de sa rivale. Il est question ici de faire appel aux services de spécialistes rituels locaux qui portent le nom générique de babaylan aux Visayas et de catalonas à Luzon. Il faut noter que ce terme de catalona est très probablement une variation phonétique sur le malais keturunan, qui signifie la «descente » de l'esprit invoqué dans le corps de la desservante à l'occasion de rituels de transe. Les entités qui « descendent » dans le corps des babaylan, et dont en vérité on ne sait trop s'il s'agit de génies locaux ou de divinités, sont généralement nommées anito dans les sources espagnoles. Ainsi le terme maganito représente-t-il une variation sur paganito, qui veut dire « entrer en relation avec les anito, solliciter les anito ».

Le troisième et dernier registre du commerce coupable avec les savoirs rituels locaux concerne le basculement vers l'islam. C'est assurément la catégorie de comportements la plus inquiétante aux yeux des agents de l'Inquisition, car il est ici question d'apostasie, de "séparation d'avec la Foi », et non pas seulement de "superstitions ", c'est-à-dire de croyances erronées mais qui n'impliquent pas une renonciation volontaire aux dogmes de l'Eglise.

14 La question du rapport des Espagnols des Philippines à l'islam malais est complexe et n'a que rarement été posée en tant que telle. Rappelons tout d'abord que dans les années 1570 et 1580, il existe, à Manille, à Mexico et à Madrid, un rêve de Chine particulièrement puissant dans l'esprit d'un certain nombre de lettrés et de conseillers du prince, c'est-à-dire de conseillers de Philippe II. L'idée que Manille puisse devenir la base arrière d'une conquête tout aussi bien militaire que spirituelle de la Chine occupe les esprits jusque tard dans le $16^{\text {ème }}$ siècle. Vous connaissez probablement la célèbre lettre que Philippe II écrit en juin 1580 à l'empereur de Chine WANLI pour l'assurer de son amitié et, dans la foulée, l'enjoindre à une conversion immédiate à la vraie Foi. Cette lettre ne fut jamais acheminée jusqu'à son destinataire, car le vice-roi de Nouvelle-Espagne et le gouverneur des Philippines craignaient - à juste titre ! - les conséquences d'une prise de contact aussi brutale. Il y a donc ce rêve de Chine, que nous retrouvons sous la plume de plusieurs missionnaires célèbres, chez les Augustins aussi bien que chez les Jésuites - je pense ici à Martin de RADA et à Alonso SANCHEZ. Le premier évêque de Manille, le dominicain Domingo de SALAZAR, espère également profiter de la présence espagnole à Manille pour faire en sorte que des membres de son ordre puissent «se faufiler » en Chine. Prenant ces rêveries pour argent comptant, de nombreux historiens contemporains tiennent pour acquis que Manille était une sorte de simple étape sur la voie de la Chine, que son histoire était tout entière déterminée par sa proximité avec la Chine.

Mais la réalité est bien différente, car en dépit des rodomontades des uns et des autres, comme celle du gouverneur Francisco de SANDE qui se targue en 1576 de pouvoir jeter à bas l'Empire des Ming avec 3000 hommes, aucune expédition militaire ne fut jamais envoyée depuis Manille à destination des côtes chinoises. En revanche, une puissante armada a été envoyée en 1578, à l'initiative du même Francisco de SANDE, à l'assaut du sultanat de Brunei, situé sur la côte nord de l'île de Bornéo. La jornada de Brunei de 1578 est une entreprise de taille conséquente, puisque SANDE avait contraint 
l'ensemble des soldats valides de la colonie à y prendre part. Pour résumer l'issue assez piteuse de cette entreprise, on peut dire qu'il s'est passé ce qui se passe presque toujours dans le monde malais lorsque des pirates vous attaquent : vous fuyez les côtes, vous vous réfugiez dans la jungle et vous attendez tranquillement que les maraudeurs repartent. L'ensemble de la noblesse brunéienne a fui l'attaque espagnole et a trouvé refuge plus en amont le long du fleuve. Les Espagnols se sont installés un peu moins de trois mois dans la capitale abandonnée et y ont contracté le paludisme et la dysenterie. Beaucoup en sont morts et les autres ont dû plier bagage sans avoir mis la main sur quoi que ce soit de précieux.

Cela vous montre à quel point, pour les Espagnols de Manille, le véritable ennemi est l'islam. C'est à la fois un ennemi extérieur, puisque l'idée prédomine que le sultan de Brunei passe son temps à fomenter des attaques pour déloger les Espagnols de leurs bases aux Philippines, mais c'est également un ennemi intime, qui ronge de l'intérieur la petite colonie espagnole. J'en veux pour preuve deux exemples tirés de dossiers inquisitoriaux, deux procès engagés à l'encontre de soldats accusés d'avoir basculé vers l'islam. Le premier procès se tient en 1582 et est instruit par l'évêque de Manille, Domingo de SALAZAR : il concerne un soldat désargenté du nom de Marcos QUINTERO. Celui-ci a gagné les Philippines en 1575 et est devenu, faute d'avoir pu bénéficier d'une encomienda, commerçant de riz. Il a épousé une Indienne et passe son temps dans les vallées de Pampanga à négocier en gros ou en demi-gros du grain dans les pueblos de Indios pour le revendre à Manille. L'accusation formulée à son encontre constitue une longue chaîne de rumeurs et de ouï-dire. Il est assigné à comparaître pour répondre surtout de deux faits. Le premier concerne les bracelets d'étain (les bitiques) qu'il porte aux chevilles et aux avant-bras "à la manière des Maures ». Ainsi, pour les jugesinquisiteurs, le basculement vers l'islam tient autant à une question d'apparence qu'à des raisons théologiques. Si on ressemble, en place publique, aux Indiens, qui sont suspectés de continuer à pratiquer clandestinement l'islam, cela constitue déjà un premier pas en direction de l'acceptation de cette foi. Cerné par les dépositions à charge, QUINTERO finit d'ailleurs par avouer que lors de l'un de ses séjours dans la maison d'un Indien, « il s'est saisi d'un guyape, qui est une vielle de maure, et en a joué, et a chanté des romances de maures qui évoquent des amours malheureuses, et qu'il portait alors au bras droit, sous son gilet et sa chemise, deux manilles d'or et s'était mis un turban sur la tête».

Le deuxième fait reproché à QUINTERO par plusieurs témoins est beaucoup plus embarrassant : il est accusé d'avoir participé à des zambras organisées par les Indiens. Cette notion de zambras, de cérémonies dansées et chantées, est d'autant plus intéressante qu'elle nous renvoie sans détour aux pratiques festives des Morisques de Grenade. Ce n'est bien sûr pas un hasard, puisque les Espagnols des Philippines sont, à l'instar de leurs compatriotes de la Nouvelle-Espagne, hantés par ce membre fantôme qu'est la présence morisque. En 1586, l'un des auditeurs de l'Audience de Manille, Melchor DAVALOS, produit un ensemble de mémoires assez copieux à l'attention du Roi, dans lesquels il établit une comparaison terme à terme entre les Maures des Philippines et les Morisques de la Péninsule, rappelle en détail les ravages consécutifs à la « grande trahison » morisque (la rébellion des Alpujarras de 1568-1571), et pour finir exhorte placidement Philippe II à ordonner, en guise de mesure préventive, l'expulsion de tous les musulmans des Philippines. 
18 Revenons-en à QUINTERO: le pire, pour l'Inquisition, reste qu'il a consciemment dissimulé, au sens ancien et religieux du terme, sa condition hybride, et qu'ainsi il a volontairement ruiné, en refusant d'endosser l'une ou l'autre des identités qu'elle démarque, la frontière séparant les mondes constitutifs de l'espace de contact colonial. Car on l'a entendu prononcer ces paroles indignes : « Je suis maure quand je me trouve chez les Maures, et chrétien quand je suis parmi les Chrétiens (quando me allo entre moros soy moro y quando entre cristianos cristiano) ». Ce que les garde-barrières de l'ordre colonial ne pardonnent pas à QUINTERO, c'est de ne pas avoir l'esprit de passeport. Ils lui infligent d'ailleurs une peine des plus sévères. Outre à assister torse dénudé et cierge en main à une messe au cours de laquelle l'officiant doit rappeler son délit, QUINTERO est condamné à recevoir 200 coups de fouet et à servir un an à l'Hôpital Royal de Manille. S'il tente de se soustraire à cette dernière obligation, c'est de 300 coups d'azote et de 4 années de galères dont il écopera.

19 Autre exemple : un procès par contumace instruit en 1588 par Domingo de SALAZAR. Cette fois, il ne s'agit plus d'un homme qui aurait basculé de manière presque inconsciente vers l'islam, en se vêtant et dansant à la manière des Indiens, mais d'un véritable renégat. L'accusé est un certain Miguel de PIŇA, ancien criado du gouverneur Santiago de VERA. PIŇA a déserté à Makassar, aux Iles Célèbes, puis s'est converti à l'islam sous le parrainage du sultan de Brunei, auprès de qui il a pris du service. Pour quelqu'un qui a une idée de ce qu'est l'islam malais-musulman de l'époque, la description de la conversion paraît totalement fantasmatique. On nous explique que Miguel de PIŇA a été entièrement rasé des mollets jusqu'au haut du crâne, puis qu'on l'a immergé tout entier dans l'eau tandis qu'il prononçait la shahada, c'est-à-dire la profession de foi musulmane. Cela correspond assez peu à ce que nous pouvons connaître de la réalité des pratiques de conversion! Le dossier nous apprend ensuite que PIŇA est devenu une sorte de conseiller militaire du sultan de Brunei, à qui il a enseigné les secrets de la fabrication des mèches d'arquebuse et de la construction de tours fortifiées capables de supporter de l'artillerie lourde. PIŇA, c'est le renégat dans tous les sens du terme, celui qui, ayant "changé de loi » au sens du passage d'une religion à une autre, a changé d'allégeance politique et s'est mis au service de l'adversaire.

On pourrait multiplier les exemples, car cette série de dossiers philippins de l'Inquisition mexicaine est particulièrement riche. Ce que l'on constate, c'est que Manille était bien une ville-mondes - un terme que je tiens à conserver au pluriel. Bien entendu, un monde nouveau a émergé du fait de la présence espagnole aux Philippines, un monde de pratiques métisses. Mais par bien des aspects, l'historicité des mondes locaux ne s'est jamais cantonnée au réduit étriqué du rapport avec les Européens. Ainsi, plusieursmondes, plusieurs univers de pratiques et de savoirs ont coexisté, entre lesquels il existait des points de passage et des zones de contact et de chevauchement, mais qui n'ont jamais coïncidé exactement les uns avec les autres. Les mondes locaux, philippins aussi bien que malais-musulman, ont conservé leur autonomie, comme le prouve l'exemple des babaylan dont les doñas rétribuent les services, et qui continuent à œuvrer dans un cadre rituel profondément cohérent et somme toute très peu affecté par la christianisation, laquelle se résume souvent à des baptêmes de masse sans catéchèse préalable. Les anito des Philippines sont donc comme les huaca des Andes : on a beau leur donner la chasse et les briser en mille morceaux, leur culte prospère à l'ombre des décombres de la conquête. 
21 De façon générale, si les Espagnols se sont rapidement convaincus qu'ils avaient pris les Philippines, s'ils ont tenté un peu plus longuement de se convaincre qu'ils avaient compris les Philippines, ils ont été eux-mêmes largement pris dans les rets des analogies sous-tendant les systèmes d'action rituels indigènes. Ainsi, pour citer la dernière et si poignante phrase de l'ouvrage de Nathan WACHTEL, "les vaincus remportent-ils, dans leur défaite, une émouvante victoire ». La question subsiste même de savoir en quel lieu, de quelle manière, et si la bataille a vraiment eu lieu. Je vous remercie de votre attention. (Applaudissements).

\section{Serge GRUZINSKI}

Merci beaucoup, Romain de nous avoir menés très loin et aux antipodes dans ce Pacifique espagnol et asiatique. Nous aborderons des contrées plus «fraîches » en écoutant un autre témoin de cette nouvelle génération, à savoir Gilles HAVARD qui est directeur de recherche au CNRS. Par ailleurs, ce dernier vient de publier son dernier ouvrage Histoire de coureurs des bois.

\section{RÉSUMÉS}

Rares sont les documentations qui nous permettent de dresser le tableau des présences espagnoles dans l'archipel philippin au cours des deux décennies suivant l'arrivée de l'armada de Miguel López de Legazpi à Cebu, en avril 1565. Dans les précis d'histoire impériale qui évoquent « le Pacifique des Ibériques », l'usage du terme de "Conquête », toujours affublé d'une majuscule, dispense ordinairement de décrire par le menu les opérations, plurielles et hasardeuses, au moyen desquelles les Espagnols édifient leurs premières enclaves fortifiées, accomplissent la «pacification » de l'île de Panay et s'emparent en 1571 de Manille, la capitale du royaume de raja Suleyman. Hormis les missives de Legazpi, les courriers des réguliers et les mémoires de supplique des capitaines - tous documents enclins aux euphémismes de bienséance -, il n'existe pratiquement aucune source permettant d'écrire une histoire sociale des "peuplements" espagnols de la fin du 16ème siècle. De précieuses informations peuvent toutefois être glanées dans les registres de la Contaduría - les livres de compte de Manille pour la période 1565-1590. Surtout, l'historien peut mettre à profit les actes d'une affaire judiciaire à tous égards exceptionnelle : le procès pour " atteinte à la Foi » intenté en 1577 par le Gouverneur Francisco de Sande au muchacho Diego de Ávila et à deux « ensorceleuses indiennes » originaires de Cebu prénommées l'une Inés, l'autre Beatriz. Au fil de nombreux témoignages éclairant le cheminement souterrain d'une rumeur d'impiété attentant à l'honneur du Gouverneur, c'est tout l'univers chamarré des ritualités et des domesticités locales qui émerge des pénombres - un univers où se côtoient, dans les dépendances des posadas et les arrière-cours des paillottes, des soldats espagnols nés au Michoacán, des guérisseuses philippines, des serviteurs malais originaires des Moluques et des Célèbes, des artisans chinois et des devins africains venus des comptoirs de l'Estado da India. L'incapacité des interprètes et du scribe du Gouverneur à traduire certains termes vernaculaires employés par Inés et Beatriz offre également la possibilité de reconstituer, par petites touches, l'univers des babaylan - les «spécialistes rituelles " qui, lorsqu'elles officient lors des cérémonies de guérison, invoquent les anito et manient de complexes pharmacopées à base de plantes, de résines et de sécrétions animales. Ici comme en 
bien d'autres situations de contact de l'époque moderne, nous n'avons donc pas affaire à la « rencontre » à huis clos entre des « cultures » étanches et homogènes, mais à la coexistence de mondes irréductibles à l'espace étriqué de leur entrecroisement.

\section{AUTEUR}

ROMAIN BERTRAND

CERI, Sciences Po/CNRS 